\title{
Dislocation Kink-Pair Energetics and Pencil Glide in Body-Centered-Cubic Crystals
}

\author{
A. H. W. Ngan $^{1}$ and M. Wen ${ }^{2}$ \\ ${ }^{1}$ Department of Mechanical Engineering, The University of Hong Kong, Pokfulam Road, Hong Kong, People’s Republic of China \\ ${ }^{2}$ Advanced Materials Section, Production Fundamentals Division, Chugoku National Industrial Research Institute, AIST, MITI, \\ Japan, 2-2-2 Hiro-suehiro, Kure, Hiroshima, 737-0197, Japan
}

(Received 3 November 2000; published 31 July 2001)

\begin{abstract}
When body-centered-cubic crystals undergo plastic deformation, the slip planes are often noncrystallographic. By performing atomistic simulation on the activation pathway of dislocation jumps in bcc iron, we show that the main reason for bcc crystals to exhibit this phenomenon is that one type of kink pair has significantly lower energy than all the other types on the same slip plane. Dislocation motion therefore cannot continue on the same slip plane, and the dislocation has to cross slip onto an intersecting slip plane after each atomic jump. Thus in the long run, the average slip plane would be zigzag and noncrystallographic.
\end{abstract}

PACS numbers: 61.72.Lk, 62.20.Fe

Compared to close-packed structures, the deformation of body-centered cubic (bcc) crystals is very unusual. First, the slip behavior in bcc crystals violates Schmid's law. The Peierls stresses of bcc metals are also 2 to 3 orders of magnitude higher than those of close-packed metals, but the flow stress decreases sharply as temperature increases. Another unique phenomenon is that, except at temperatures approaching absolute zero, the slip lines are wavy or diffuse, and they align, on average, along the maximum resolved shear stress plane, which can be noncrystallographic. This phenomenon, suggested initially by Taylor [1], is widely known as "pencil glide."

These unusual characteristics have attracted a large volume of 2D atomistic simulations, first due to Duesbury, Vitek, and coworkers [2], on the characteristics of the ground state configuration and motion of screw dislocations. The concepts derived from these investigations have now become classical, and the relevant literature has been reviewed by several authors [3]. These studies have led to a satisfactory explanation of the high Peierls stress and the non-Schmid behavior of the bcc structure. A lot of the 2D atomistic results have also indicated that, because of the dissociation nature of the dislocation core, the motion of $\frac{1}{2}\langle 111\rangle$ screw dislocations would in general follow zigzag paths [3]. While this has formed a good basis for the explanation of pencil glide, the fact that straight dislocations were simulated in these studies weakens the extension of the predictions to finite temperatures, at which it is widely accepted that the motion of sessile dislocations should take place by the kink-pair mechanism $[4,5]$.

In an attempt to understand the dependence of yield strength on temperature, we have recently carried out atomistic simulations to determine the activation energetics of the kink-pair mechanism of $\frac{1}{2}\langle 111\rangle$ screw dislocations in bcc iron [6]. In this Letter, we will focus on establishing the link between kink-pair energetics and pencil glide. In $\alpha$ iron, the ground state of the $\frac{1}{2}\langle 111\rangle$ screw dislocation is twofold degenerate. The two degenerate states are diad related about the screw axis as shown in Figs. 1(b) and 1(d), and are referred to as the $B$ and $A$ states, respectively. Depending on the states before and after a unit step of dislocation advancement, a number of kink-pair configurations are thus possible. We use here the Duesbury scheme of nomenclature [7], in which the kink pair arising from the jump along, for example, the negative Peach-Koehler force direction from a $B$ state to an $A$ state is denoted as the $\mathrm{Bn} A \mathrm{p} B$ kink pair (Fig. 1). In this example, on going along the positive dislocation line direction, the first kink $B \mathrm{n} A$ has the kink segment pointed along the negative Peach-Koehler force direction, while the second kink $A \mathrm{p} B$ has the kink segment pointed along the positive Peach-Koehler force direction. In general, there are thus eight possible kink-pair configurations, namely, $A \mathrm{n} B \mathrm{p} A, B \mathrm{n} A \mathrm{p} B, A \mathrm{n} A \mathrm{p} A$, and $B \mathrm{n} B \mathrm{p} B$ operating under a negative Peach-Koehler force, and $A \mathrm{p} B \mathrm{n} A$, $B \mathrm{p} A \mathrm{n} B, A \mathrm{p} A \mathrm{n} A$, and $B \mathrm{p} B \mathrm{n} B$ operating under a positive Peach-Koehler force. However, from symmetry arguments [6], these form equivalent pairs as shown in Table I, so that we need only to consider, for example, the $A \mathrm{n} B \mathrm{p} A$, $B \mathrm{n} A \mathrm{p} B, A \mathrm{n} A \mathrm{p} A$, and $B \mathrm{n} B \mathrm{p} B$ nonequivalent configurations operating under a negative Peach-Koehler force.

To locate the minimum energy paths (MEP) of the formation and expansion of the kink pairs, we have used the nudged elastic band method [8,9]. In this method, replicas of the system are initially placed equispaced along an initially chosen MEP connecting the reactant and product states. Each replica is then relaxed perpendicular to the original MEP to obtain a better MEP, and the process is iterated a number of times to obtain a good estimate of the true MEP. In our simulation, we have used Johnson and Oh's embedded atom potential for iron [10] to represent the atomic interaction in each replica. This potential produces threefold dissociated ground states of the $A$ and $B$ type as shown in Figs. 1(b) and 1(d). To achieve quicker convergence, an arbitrary kink-pair shape was introduced into the initial MEP. Relaxation of the replicas was carried out using a molecular dynamics code on an IBM SP2 parallel computer, with each processor handling a few replicas. 

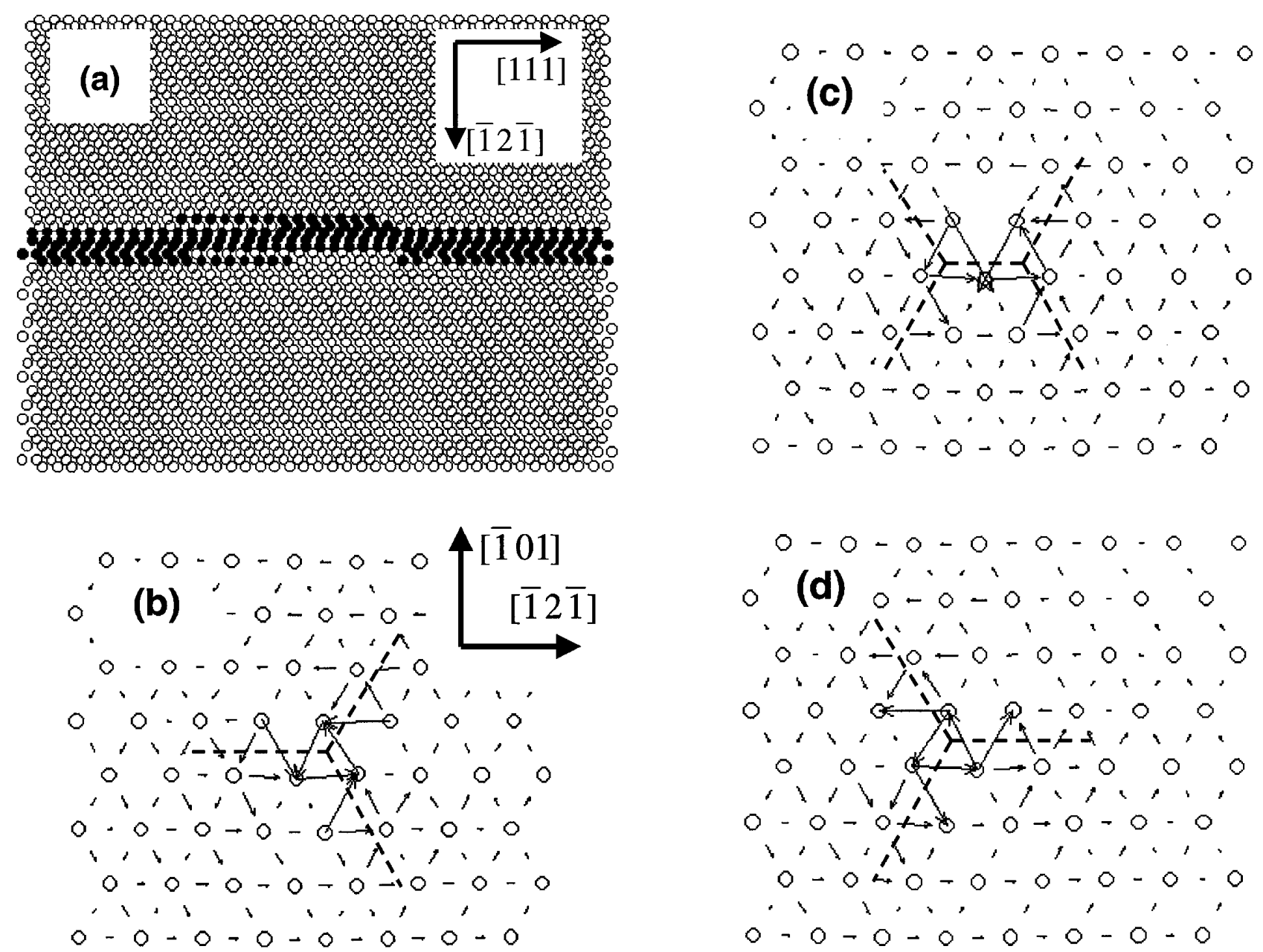

FIG. 1. (a) Saddle configuration of the $B \mathrm{n} A \mathrm{p} B$ kink pair in a $\frac{1}{2}\langle 111\rangle$ screw dislocation at $320 \mathrm{MPa}$. The two $\{101\}$ slip planes sandwiching the dislocation are shown. The filled atoms are those with energies higher than $4.22 \mathrm{eV}$, and so they trace out the dislocation line. Here, the left and right segments are in the reactant " $B$ " state, while the central kink-span segment is in transition to the product " $A$ " state. (b) -(d) Differential displacement maps of the dislocation core when viewed end-on, showing the reactant " $B$ " state (b), the kink span of the saddle state (c), and the product " $A$ " state (d). Each arrow in (b)-(d) marks the relative displacements along [111] between two atoms. The dotted lines show roughly the dissociation planes.

Typically, 16 replicas were relaxed with 8 processors, and relaxation was done by 2000 molecular dynamics steps following quenching from an equilibrated temperature of, say, $15 \mathrm{~K}$ to absolute zero. The typical residual interatomic forces were smaller than $10^{-4} \mathrm{eV} / \AA$. The supercell size used in the simulation was typically 11 repeated cells along the $[1 \overline{2} 1]$ direction, 19 repeated cells along the [ $\overline{1} 01]$ direction, and 40 Burgers vectors along the [111] direction. The Burgers vector was chosen as $\frac{1}{2}[\overline{1} \overline{1} \overline{1}]$ when viewed along the [111] direction. The periodic boundary condition was applied along [111], while the (12)1) and (101) faces were kept as free surfaces. Stresses of the desired magnitudes and along the Burgers vector direction were applied on the (101) faces. Occasional checks were performed on

TABLE I. Symmetry of kink pairs. The two kink pairs in each row are diad related about the slip plane normal and behave the same under opposite stresses.

\begin{tabular}{cc}
\hline \hline $\begin{array}{c}\text { Kink pair operating under } \\
\text { positive Peach-Koehler force }\end{array}$ & $\begin{array}{c}\text { Equivalent kink pair operating under } \\
\text { negative Peach-Koehler force }\end{array}$ \\
\hline$A \mathrm{p} B \mathrm{n} A$ & $B \mathrm{n} A \mathrm{p} B$ \\
$B \mathrm{p} A \mathrm{n} B$ & $A \mathrm{n} B \mathrm{p} A$ \\
$A \mathrm{p} A \mathrm{nn} A$ & $B \mathrm{n} B \mathrm{p} B$ \\
$B \mathrm{p} B \mathrm{n} B$ & $A \mathrm{n} A \mathrm{p} A$ \\
\hline \hline
\end{tabular}


supercells that were one time longer along [111], but almost identical results were obtained. This confirms that the supercell was long enough to eliminate interactions between adjacent supercells introduced by the periodic boundary condition.

Of the four possible nonequivalent kink pairs corresponding to jumps along the negative Peach-Koehler force direction, we find that the $B \mathrm{n} A \mathrm{p} B$ is the one with significantly lower activation energy as shown in Fig. 2 [11]. This suggests that, under a negative Peach-Koehler force, the $B$ to $A$ jump is far easier than all other possible jumps [12]. The next question is what will follow this easy $B$ to $A$ jump, bearing in mind that both the $A$ to $B$ jump (involving the $A \mathrm{n} B \mathrm{p} A$ kink pair) and the $A$ to $A$ jump (involving $A \mathrm{n} A \mathrm{p} A$ ) have much higher activation energies. As motion forward is difficult on the same slip plane, an option would be for the $A$ state to cross slip onto an intersecting $\{101\}$ plane to form a $B$ state. Let us denote the associated kink pair as $A \mathrm{n}(B) \mathrm{p} A$, where $(B)$ means that the state $B$ is in a cross-slip plane. In simulating the cross-slip kink pair, the nearest free boundary will exert an additional driving force on the dislocation jump but trial runs using a doubled block size showed that this effect is small on the activation energies. Figure 3(a) shows the atomic configuration of the activated state kink-span portion of such a kink pair. The energies of the $A \mathrm{n}(B) \mathrm{p} A$ kink pair are also shown in Fig. 2, and we see that the $A$ to $(B)$ cross-slip jump also has similar energy or is as easy as the $B$ to $A$ jump. Hence we conclude that the lowest energy path, at stress levels below the Peierls stress, should involve the $B$ to $A$ jump, followed by the $A$ to $(B)$ cross-slip jump, and then the sequence of slip and cross slip may repeat. Since at a given $A$ state, cross slip can happen onto either of the two intersecting $\{101\}$ planes, it can be expected that in the long

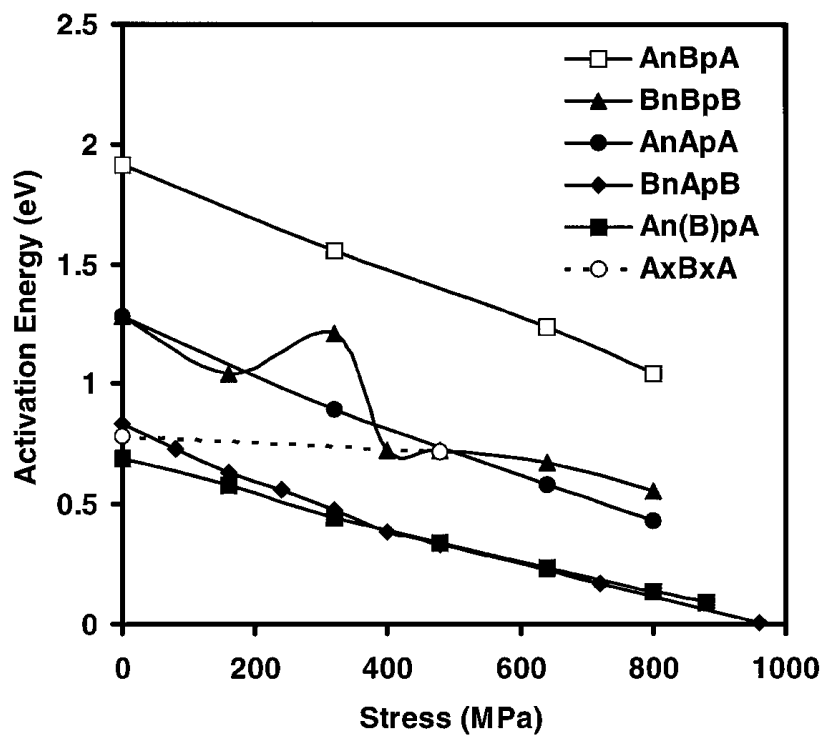

FIG. 2. Activation energy vs stress for all kink pairs and the $A \times B \times A$ flip pair. run, the average slip plane would tend toward the maximum resolved shear stress plane. This is how pencil glide happens.

The activation pathways of the $B \mathrm{n} A \mathrm{p} B$ and the $A \mathrm{n}(B) \mathrm{p} A$ kink pairs involve kink-pair formation and expansion at all stresses up to the Peierls stress, at which no stable ground state can be found. A typical kink-pair configuration is shown in Fig. 1. Figure 3(b) shows the activation barriers at increasing stress of $A \mathrm{n}(B) \mathrm{p} A$; those of $B \mathrm{n} A \mathrm{p} B$ are similar. In accordance with classical line-tension models $[4,5]$, both the kink span and the activation energy drop with increasing stress. The activation energies of these two kink pairs are low because in the saddle configuration, the inclining arms of the $A$ and $B$ states are kept apart [see Figs. 1(c) and 3(a)]. Since most of the Burgers vector content of the screw dislocation is stored in the dissociated planes, keeping the dissociated arms of the $A$ and $B$ states apart would result in a low interaction energy. The activation pathways of the other high energy kink pairs involve high energy kink configurations. In the saddle configuration of the $A \mathrm{n} B \mathrm{p} A$ kink pair, the inclining arms intersect to produce a high energy configuration as shown in
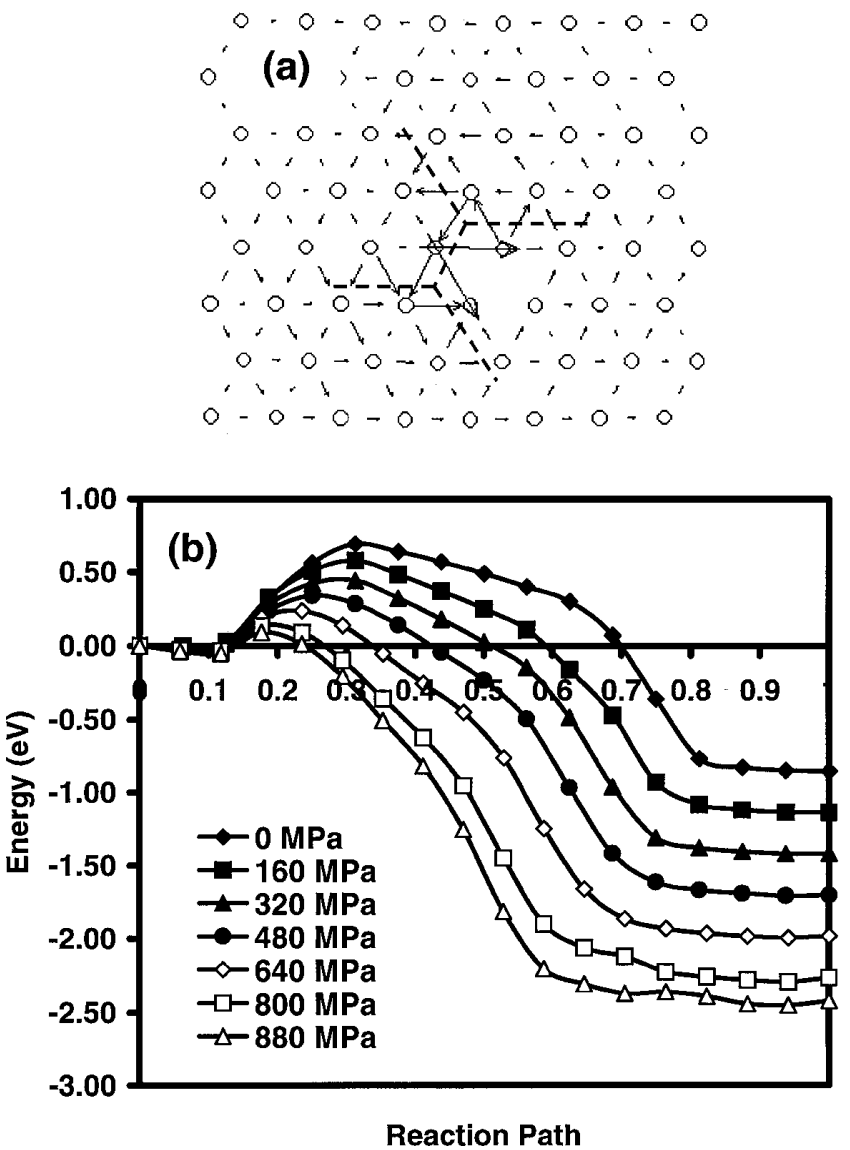

FIG. 3. The $A \mathrm{n}(B) \mathrm{p} A$ cross-slip kink pair. (a) The configuration of the kink span of the saddle state of $A \mathrm{n}(B) \mathrm{n} A$ at $320 \mathrm{MPa}$, showing the moment when the $A$ core is about to cross slip to form a $B$ core along an intersecting slip plane. (b) Activation barriers at increasing stress of the $A \mathrm{n}(B) \mathrm{p} A$ kink pair. 


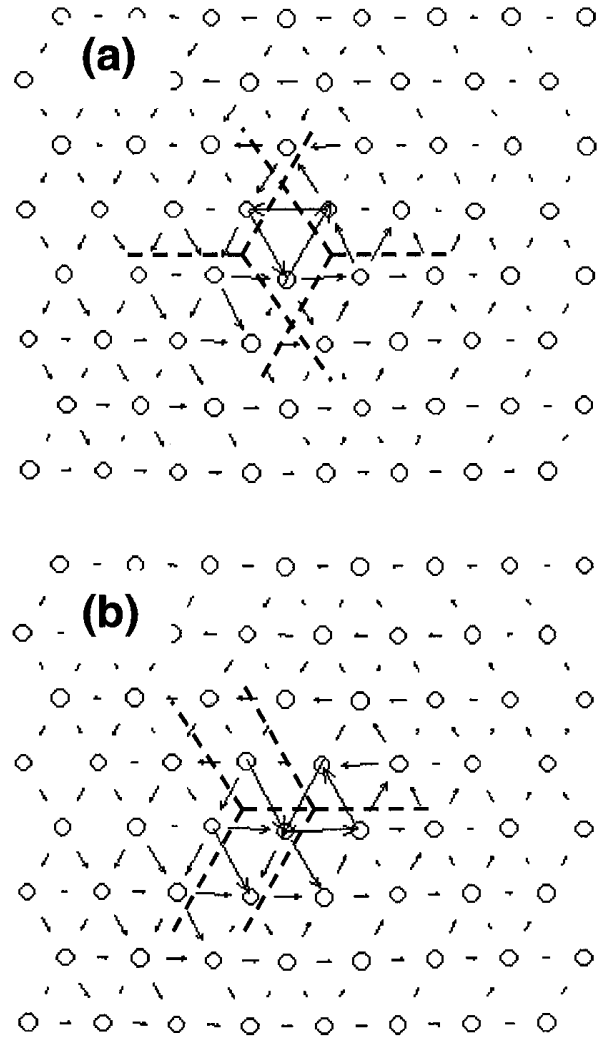

FIG. 4. Differential displacement maps of the saddle state kink-span region of the $A \mathrm{n} B \mathrm{p} A$ kink pair (a) and the $A \mathrm{n} A \mathrm{p} A$ kink pair (b), both at $800 \mathrm{MPa}$.

Fig. 4(a), while in the saddle configuration of the $A \mathrm{n} A \mathrm{p} A$ kink pair, the inclining arms of one state overlay on those of the other as shown in Fig. 4(b). The $B \mathrm{n} B \mathrm{p} B$ kink pair is found to always decompose into two steps, comprising first the easy $B \mathrm{n} A \mathrm{p} B$ kink pair, followed by a flip of the kink span from state $A$ back to state $B$ in the same Peierls valley. At stresses smaller than $400 \mathrm{MPa}$, the $B \mathrm{n} A \mathrm{p} B$ kink pair and the "flip" occur simultaneously so that the activation energy is quite high (see Fig. 2). At larger stresses, the $B \mathrm{n} A \mathrm{p} B$ kink pair is well formed and expanded before the "flip" nucleates within the kink span. The activation of the whole process is then dominated by that of the "flip." In Fig. 2 is also shown the activation energy of the $A \times B \times A$ "flip pair," where " $x$ " denotes the flip defect on the dislocation line. It can be seen that the flip-pair energy stays roughly constant at a value of about $0.7 \mathrm{eV}$ as stress increases. The activation energy of the flip is not so much affected by stress because the dislocation remains in the same Peierls valley during flip so that the stress does little work.
In the above considerations, we have focused only on the energies of isolated kink pairs. In reality in a screw dislocation line, thermal fluctuations may produce random segments of both the diad states connected by flip defects. Since the $B \mathrm{n} A \mathrm{p} B$ and the $A \mathrm{n}(B) \mathrm{p} A$ kink pairs have very similar activation energies, under a negative applied stress (for example), the $B$ segment will jump forward on the same plane, while the $A$ segment will cross slip, i.e., the initially different segments will develop their own jump paths. The initial flip defects will soon develop into edge dipole barriers, but these may be annihilated dynamically. As for the question of whether dynamic flipping would affect dislocation jumps, Fig. 2 shows that at finite stresses, the flip pair has far higher activation energies than the easy kink pairs $B \mathrm{n} A \mathrm{p} B$ or $A \mathrm{n}(B) \mathrm{p} A$. This means that, at finite stresses, dislocation jumps would occur far more frequently than dynamic flip defect formation.

A. H. W. N. is grateful to L.P. Kubin for helpful discussion. This research was supported by a research grant (No. HKU 572/96E) from the Research Grants Council, Hong Kong Special Administrative Region of the People's Republic of China.

[1] G. I. Taylor and C. F. Elam, Proc. R. Soc. London A 112, 337 (1926).

[2] M. S. Duesbury, V. Vitek, and D. K. Bowen, Proc. R. Soc. London A 332, 85 (1973).

[3] For reviews, see V. Vitek, Cryst. Lattice Defects 5, 1 (1974); M.S. Duesbury, in Dislocations in SolidsBasic Problems and Applications, edited by F. R. N. Nabarro (North-Holland, Amsterdam, 1989), Vol. 8, pp. 67-173; T. Suzuki, S. Takeuchi, and H. Yoshinaga, Dislocation Dynamics and Plasticity (Springer, Berlin, 1991), Chap. 6

[4] J. E. Dorn and S. Rajnak, Trans. AIME 230, 1052 (1964).

[5] M. S. Duesbury, Philos. Mag. 19, 501 (1969).

[6] M. Wen and A. H. W. Ngan, Acta Mater. 48, 4255 (2000).

[7] M. S. Duesbury, Acta Metall. 31, 1747 (1983).

[8] G. Mills, H. Jónsson, and G. K. Schenter, Surf. Sci. 324, 305 (1995).

[9] T. Rasmussen et al., Phys. Rev. Lett. 79, 3676 (1997).

[10] R. A. Johnson and D. J. Oh, J. Mater. Res. 5, 1195 (1989).

[11] The energy of a kink pair is the simulated configurational energy minus the work done by the applied stress. The work done is calculated from the displacements of the atoms on the stressed surfaces of the supercell.

[12] The $A$ to $B$ jump involving the $A \mathrm{p} B \mathrm{n} A$ kink pair will be the easiest along the positive Peach-Koehler force direction. 